\title{
The nonlinear behavior of a sheared immiscible fluid interface
}

\author{
Warren Tauber and Salih Ozen Unverdi \\ Department of Mechanical Engineering, University of Michigan, Ann Arbor, Michigan 48109
}

\author{
Gretar Tryggvason ${ }^{\text {a) }}$ \\ Department of Mechanical Engineering, Worcester Polytechnic Institute, Worcester, Massachusetts 01609
}

(Received 9 July 2001; accepted 23 April 2002; published 3 July 2002)

\begin{abstract}
The two-dimensional Kelvin-Helmholtz instability of a sheared fluid interface separating immiscible fluids is studied by numerical simulations. The evolution is determined by the density ratio of the fluids, the Reynolds number in each fluid, and the Weber number. Unlike the KelvinHelmholtz instability of miscible fluids, where the sheared interface evolves into well-defined concentrated vortices if the Reynolds number is high enough, the presence of surface tension leads to the generation of fingers of interpenetrating fluids. In the limit of a small density ratio the evolution is symmetric, but for a finite density difference the large amplitude stage consists of narrow fingers of the denser fluid penetrating into the lighter fluid. The initial growth rate is well predicted by inviscid theory when the Reynolds numbers are sufficiently high, but the large amplitude behavior is strongly affected by viscosity and the mode that eventually leads to fingers is longer than the inviscidly most unstable one. (c) 2002 American Institute of Physics.
\end{abstract}

[DOI: $10.1063 / 1.1485763$ ]

\section{INTRODUCTION}

The Kelvin-Helmholtz instability of an initially flat interface separating two fluids moving in the opposite direction is one of the classical problems in fluid mechanics. In its simplest form, the fluids are assumed to be inviscid and the flow irrotational on either side of the interface. Linear stability analysis dates back to the nineteenth century and computations of the nonlinear formation of a concentrated vortex are among the earliest examples of computational fluid dynamics studies. ${ }^{1}$ As computers became widely available, studies progressed along two paths. Several investigators examined the evolution of an infinitely thin vortex sheet, separating two potential flow regions. This turned out to be a difficult task due to the ill-posedness of the vortex sheet problem (and the rapid growth of small perturbations) as well as the formation of a singularity at the point where the vortex was expected to appear. The difficulty was eventually resolved by Krasny ${ }^{2}$ who showed that a regularization of the vortex sheet resulted in both a well-posed problem and the elimination of the singularity formation. Other investigators examined the nonlinear evolution of the full Navier-Stokes equations (see, for example, Refs. 3 and 4) and showed that a perturbed shear layer develops into a row of vortices. Tryggvason, Dahm, and Sbeih ${ }^{5}$ examined the limit of high Reynolds numbers and a small initial thickness and compared full Navier-Stokes simulations with the regularized inviscid model of Krasny. ${ }^{2}$ They showed that while it was not possible to equate the regularization directly to a physical effect, the solution appeared to approach the same limit when the

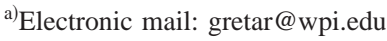

initial thickness was reduced and the Reynolds number increased, as the solution to the inviscid model when regularization was decreased.

The Kelvin-Helmholtz instability of two identical fluids is now a well understood problem (for early threedimensional simulations see Ref. 6, for example). The corresponding problem for the instability and breakup of fluids with a finite density and viscosity stratification, particularly when the fluids are immiscible and surface tension must be included, is less understood. The effect of surface tension for fluids of equal densities was examined by Hou, Lowengroub, and Shelly, ${ }^{7}$ who assumed that viscous effects could be ignored completely. They found that large surface tension suppressed roll-up and that the interface instead evolved into long fingers of interpenetrating fluids. When surface tension was lowered, the fingers folded over and in the limit of small surface tension the interface rolled up as in the classical nonlinear Kelvin-Helmholtz instability. Pozrikidis ${ }^{8}$ examined the other limit of Stokes flows and also found that the interface can develop elongated fingers and that an increase in the viscosity ratio can destabilize the interface. Both inertia and viscosity, as well as surface tension, has been included by Lafaurie, Nardone, Scardovelli, Zaleski, and Zanetti ${ }^{9}$ who also found the development of long fingers at finite Reynolds numbers. These authors examined the effect of three dimensionality and found that the two-dimensional fingers that develop initially can evolve into fully three-dimensional fingers of one fluid pointing into the other fluid.

The importance of understanding the breakup of an interface separating immiscible fluids of different material properties is considerable. Liquid fuels are usually burned by first atomizing a fuel jet to increase the surface area and hence the evaporation rate. In the prediction of spray behavior, the initial atomization is both the most critical and the 
least understood aspect of the spray. The importance of the initial breakup is well demonstrated by the large number of atomizers that have been proposed. See, for example, the books by Lefebvre ${ }^{10}$ and Bayvel and Orzechowski. ${ }^{11}$

Fluid jets break up in different ways, depending on the governing nondimensional parameters. When capillary effects are large, a jet undergoes a Rayleigh instability due to waves longer than its diameter and breaks up into a stream of relatively large drops. When capillary effects are small the jet is unstable to shorter waves that are generally enhanced by aerodynamic effects, resulting in smaller drops. At very large Weber numbers the jet breaks up into ligaments (or "fibers") that then break up into drops much smaller than the jet diameter. Under atmospheric conditions, where the external flow has little effect, the jet breakup is believed to be strongly affected by the turbulence level in the jet. However, for high pressure combustors, the density ratio is often much smaller, and the injected jet laminar. For experimental investigations of the different breakup modes see, for example, Refs. 12 and 13.

Here, we investigate the initial breakup of an immiscible fluid interface by numerical simulations of the twodimensional Navier-Stokes equation. This study extends the work of Lafaurie et al. and Hou et al. by examining the nonlinear behavior of the two-dimensional shear instability of immiscible fluids with large but finite Reynolds numbers as well as finite density differences. Limited preliminary results for two-dimensional shear layers have been reported in Refs. 14 and 15 . The second reference also included preliminary three-dimensional simulations.

\section{PROBLEM FORMULATION AND NUMERICAL METHOD}

We examine an initially nearly flat interface separating two different fluids. The fluid below the interface is moving to the right with velocity $U_{1}$ and the fluid on the top is moving to the left with velocity $U_{2}$. The density and viscosity of the bottom and top fluids are $\rho_{1}, \mu_{1}$ and $\rho_{2}, \mu_{2}$, respectively. The rectangular computational domain, sketched in Fig. 1, has periodic horizontal boundaries and rigid, moving walls at the top and the bottom. The domain size is the wavelength, $\lambda$, in the horizontal direction and $2 \lambda$ in the vertical direction. The evolution of the interface is determined by the velocity difference across the interface, the surface tension, and the density and viscosity of both fluids. When the viscosities are low enough, we expect the initial growth rate to be well predicted by linear stability analysis of inviscid flows (see Ref. 16, for example). For perturbations of the form

$$
A=A_{0} e^{s t+i k x}
$$

it is found that

$$
\begin{aligned}
s= & -i k \frac{\rho_{1} U_{1}+\rho_{2} U_{2}}{\rho_{1}+\rho_{2}} \\
& \pm \sqrt{\frac{k^{2} \rho_{1} \rho_{2}\left(U_{1}-U_{2}\right)^{2}}{\left(\rho_{1}+\rho_{2}\right)^{2}}-\frac{T k^{3}}{\rho_{1}+\rho_{2}}},
\end{aligned}
$$

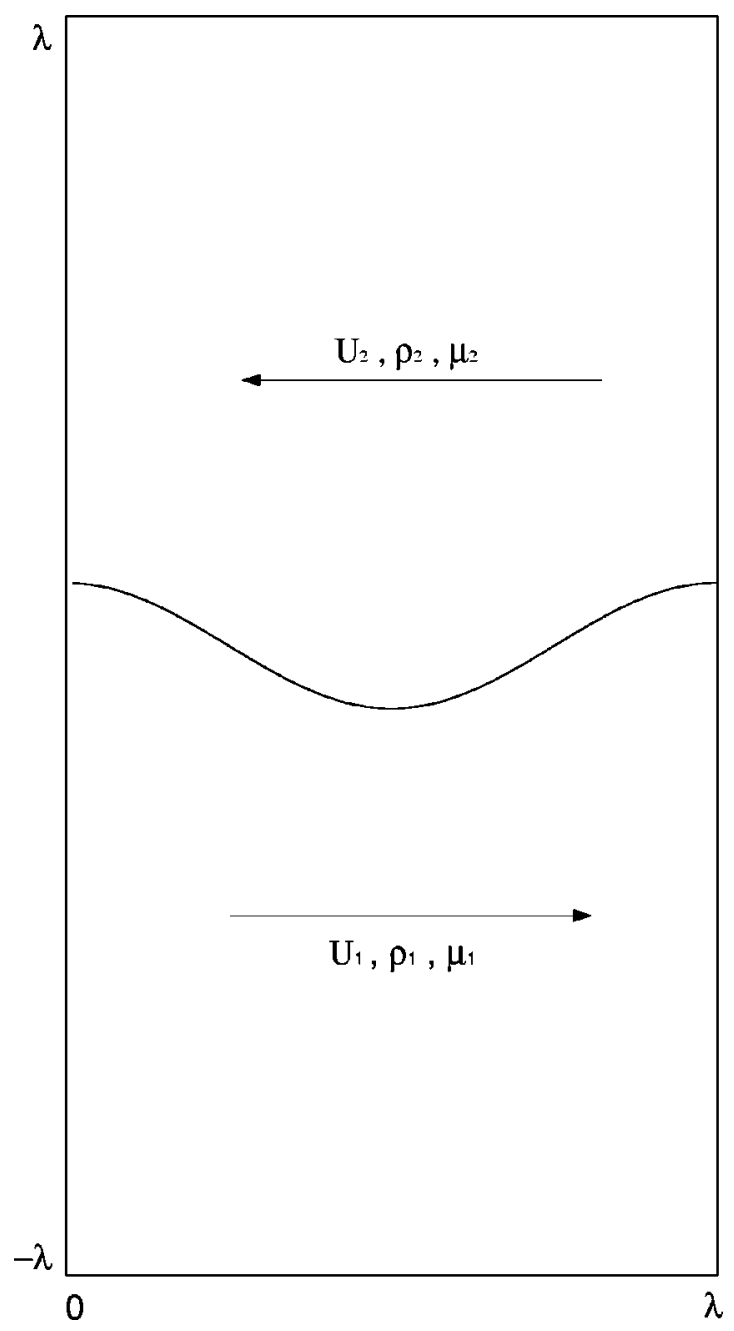

FIG. 1. Computational setup.

where $T$ is the surface tension and $k$ is the wave number. The first part of this expression is the phase velocity, $c$ $=-\operatorname{Im}(s) / k$ and the second part is the growth rate $\sigma$ $=\operatorname{Re}(s)$. In nondimensional form we have

$$
\tilde{\sigma}=\frac{\sigma T}{\rho_{2} \Delta U^{3}}=\frac{1}{\mathrm{We}} \sqrt{\frac{1}{r+1}\left(\frac{r}{1+r}-\frac{1}{\mathrm{We}}\right)}
$$

and

$$
\tilde{c}=\frac{c}{\Delta U}=\frac{1}{\Delta U} \frac{r U_{1}+U_{2}}{(r+1)} .
$$

Here we have constructed a time and a velocity scale by

$$
\tilde{\tau}=\frac{1}{k \Delta U} \text { and } \widetilde{U}=\Delta U,
$$

and introduced $\Delta U=U_{2}-U_{1}$ and $r=\rho_{1} / \rho_{2}$. The Weber number is defined as

$$
\mathrm{We}=\frac{\rho_{2} \Delta U^{2}}{T k} .
$$


The expression for the phase velocity simply shows that the initial wave is advected by the density weighted average velocity. The growth rate is real if the expression under the square root is positive, or

$$
\mathrm{We}>1+\frac{1}{r} \text {. }
$$

The growth rate is maximum for

$$
\mathrm{We}_{\max }=\frac{3}{2}\left(1+\frac{1}{r}\right)
$$

and the minimum Weber number for a real growth rate (the critical Weber number) is

$$
\mathrm{We}_{\text {crit }}=1+\frac{1}{r} \text {. }
$$

We also introduce the Reynolds numbers based on the properties of the bottom and the top fluids, respectively, as

$$
\operatorname{Re}_{1}=\frac{\rho_{1} \Delta U \lambda}{\mu_{1}} \text { and } \operatorname{Re}_{2}=\frac{\rho_{2} \Delta U \lambda}{\mu_{2}}
$$

The numerical method used for the computations presented here is based on writing one set of equations for the entire computational domain, independently of how many different fluids are involved. This is possible by allowing for different material properties in the formulation and adding singular terms at the boundaries between the different fluids to ensure that the correct interface conditions are satisfied. The resulting "one-field" Navier-Stokes equations are

$$
\begin{aligned}
\frac{\partial \rho \mathbf{u}}{\partial t}+\nabla \cdot \rho \mathbf{u u}= & -\nabla p+\nabla \cdot \mu\left(\nabla \mathbf{u}+\nabla \mathbf{u}^{T}\right) \\
& +T \int_{F}(\mathbf{n} \times \nabla) \times \mathbf{n} \delta\left(\mathbf{x}-\mathbf{x}_{f}\right) d a .
\end{aligned}
$$

Here, $\mathbf{u}$ is the velocity vector, $p$ is the pressure, $\rho$ and $\mu$ are the discontinuous density and viscosity fields, respectively, and $\mathbf{n}$ is the surface normal. The surface forces act only on the interface between the fluids and appears in the current formulation multiplied by a two-dimensional delta function, $\delta$. The integral is over the entire front or interface. This equation contains no approximations beyond those in the Navier-Stokes equations and in particular, it implicitly contains the proper stress conditions for the fluid interface. Since the density and the viscosity are different for each fluid, it is necessary to track the evolution of these fields by equations of state, which specify that each fluid particle retains its original density and viscosity,

$$
\frac{D \rho}{D t}=0 \text { and } \frac{D \mu}{D t}=0 .
$$

The momentum equation is also supplemented by an equation of mass conservation, which for incompressible flows is

$$
\nabla \cdot \mathbf{u}=0
$$

The momentum equation is discretized on a regular staggered grid using second-order, centered finite differences for the spatial derivatives and a second-order predictor- corrector time integration scheme. The continuity equation, when combined with the momentum equation results in a pressure equation that is not separable as for homogeneous flow and is solved by a multigrid method. ${ }^{17}$ To advect the material properties, and to evaluate the surface tension term in the momentum equation, we track the interface between the different phases explicitly by connected marker points (front). The number of points representing the front is selected such that there are approximately 2-4 front points per stationary mesh. As the front deforms, points are added and deleted dynamically to maintain adequate resolution. The $\delta$ function is regularized by distributing the surface force and the density gradient onto the fixed grid used to solve the momentum equation. In the computations reported here, we have used a distribution function introduced by Peskin, ${ }^{18}$ which smooths the $\delta$ function to the nearest nine grid points. The one-field formulation used here is common to other techniques for multifluid flows such as the VOF (volume of fluid) method and the more recent level set method. In these methods, however, the phase boundary is not tracked explicitly, but reconstructed from a marker function. Explicitly tracking the interface avoids the difficulty of advecting such marker functions and allows accurate evaluation of the surface tension. While very high surface tension can sometimes cause unphysical parasitic currents as well as stiffness problems, explicit tracking as well as a semi-implicit treatment of the surface tension helps minimize these problems. ${ }^{19}$

The method has been applied to a number of multifluid problems and tested and validated in a number of ways, not only to check the implementation, but also its accuracy. Those tests include comparisons with analytical solutions for simple problems, other numerical computations, and experiments. The actual resolution requirement varies with the parameters of the problem. High Reynolds numbers, for example, generally require finer resolution than lower ones, as in other numerical calculations. However, in all cases we have found that the method converges rapidly under grid refinement, and in those cases where other solutions exist we have found excellent agreement, even for modest resolutions. For a more detailed description of the method, see Ref. 19. Validations by comparison with analytical results can be found in Nobari, Jan, and Tryggvason ${ }^{20}$ and Esmaeeli and Tryggvason. $^{21}$

\section{RESULTS}

The shear instability of an initially flat interface separating two immiscible fluids moving in the opposite directions is examined here for two density ratios: $r=1$ (where both fluids have the same density) and $r=10$. This selection of density ratios is motivated by considerations of high-pressure sprays where the density difference is much smaller than at atmospheric pressures.

\section{A. Linear stability}

The velocity field is initially discontinuous and the Reynolds numbers are high, so we expect the initial growth to be well predicted by inviscid analysis [Eq. (2)]. To examine if this is the case, several simulations were done using the 
eigenmode from the stability analysis as the initial condition. To find the initial velocity field, the vortex sheet strength is determined by subtracting the perturbation velocities at the interface. In nondimensional form, the result is

$$
\tilde{\gamma}=\gamma \frac{k T}{\rho_{2} \Delta U^{3}}=-\frac{1}{\mathrm{We}}+\frac{r-1}{r+1} \frac{\tilde{\zeta}}{\mathrm{We}} \pm 2 \widetilde{\zeta} \tilde{\sigma} \sin (\tilde{x}) .
$$

Here $\widetilde{\zeta}=k \zeta$ is the nondimensional initial amplitude, $\widetilde{\sigma}$ is the linear growth rate, given by Eq. (3), and $\tilde{x}=k x$ is the nondimensional horizontal space coordinate. Given the vortex sheet strength, the circulation, $\Gamma$, at discrete points is found by integrating over a small segment of the interface:

$$
\Gamma=\int_{\Delta S} \gamma d s
$$

Once the circulation at discrete interface points has been found, it is distributed to the fixed grid next to the interface, using Peskin's distribution function, ${ }^{18}$ to give a grid-vorticity field, $\omega$. The stream function is then found by solving

$$
\nabla^{2} \psi=-\omega
$$

and the velocity computed by a centered difference approximation of

$$
u=\frac{\partial \psi}{\partial y} \quad \text { and } \quad v=-\frac{\partial \psi}{\partial x} .
$$

When the initial velocity field has been found, the solution can be integrated forward in time.

To estimate the nondimensional linear growth rate, we rewrite Eqs. (1) and (3) as

$$
\tilde{\sigma}=\frac{T}{\rho_{2} \Delta U^{3}} \frac{1}{t} \ln \left(A(t) / A_{0}\right)
$$

and plot $\tilde{\sigma}$ as a function of time. In Fig. 2, $\widetilde{\sigma}$ is shown for a Weber number equal to 1.65 , a density ratio equal to 10 , $\operatorname{Re}_{1}=\operatorname{Re}_{2}=5000$, and three different grid resolutions. $\zeta$ is taken to be $2.5 \%$ of the wavelength. If the growth rate remained constant, the line would be parallel to the time axis and equal to the solid horizontal line which is the nondimensional growth rate derived from linear inviscid theory. It is clear that the numerical computations are converging to this line at early times but as the amplitude of the wave increases, nonlinear effects reduce the growth. We have repeated these calculations for several different Reynolds numbers and find almost no changes for values ranging from 600 to 10000 , suggesting that viscous effects are indeed small at the earliest times.

In Fig. 3 the initial computed growth rate, normalized by the maximum linear inviscid growth rate, is plotted versus the inverse Weber number normalized by the critical Weber number, for $\operatorname{Re}_{1}=\mathrm{Re}_{2}=5000$ and a density ratio equal to 10 . The growth rate predicted by linear stability theory is shown by a solid line. Open circles represent grid resolution of $256 \times 512$ points, crosses are resolution of $64 \times 128$ grid points. The growth rate is well predicted, although the finite resolution used leads to a slight underprediction. Similar results are obtained for the zero stratification case. ${ }^{14}$

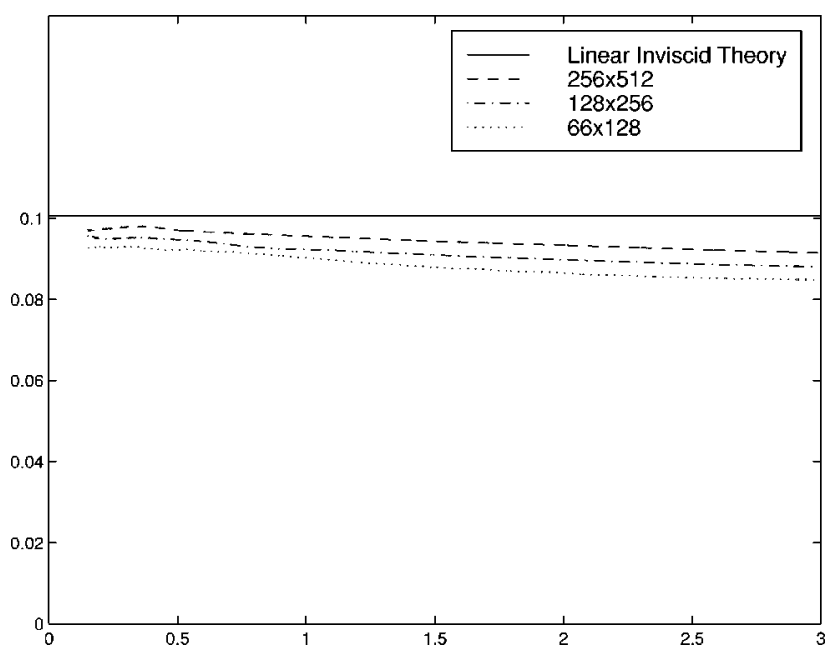

FIG. 2. Initial nondimensional growth rate vs time for density ratio $=10.0$, $\mathrm{We}=1.65, \mathrm{Re}_{1}=\mathrm{Re}_{2}=5000$.

\section{B. Nonlinear evolution for a density ratio of 1}

The evolution of an interface separating two fluids of equal densities and viscosities is shown in Fig. 4. The Weber number is equal to 3.0 , which corresponds to a wavelength equal to the linear, inviscid, most unstable one. Here, $\operatorname{Re}_{1}$ $=\operatorname{Re}_{2}=10000$ and the grid resolution is $256 \times 512$ points. The initial conditions are again derived by normal mode analysis, using $\zeta$ equal to $5 \%$ of the wavelength. The interface and stream function contours are plotted on the left, and the interface and vorticity contours are plotted on the right, for six different times.

The initial disturbance grows rapidly and the wave becomes steeper on the downwind side. First, the vorticity at the interface is advected toward the middle of the steep part in the same way as the vorticity on an interface with zero surface tension. Then, however, the vorticity peak divides in two as the interface becomes steeper. This is also seen in the inviscid simulations of Hou et al. ${ }^{7}$ In the inviscid simulations the interface continually grows but, in our finite Reynolds

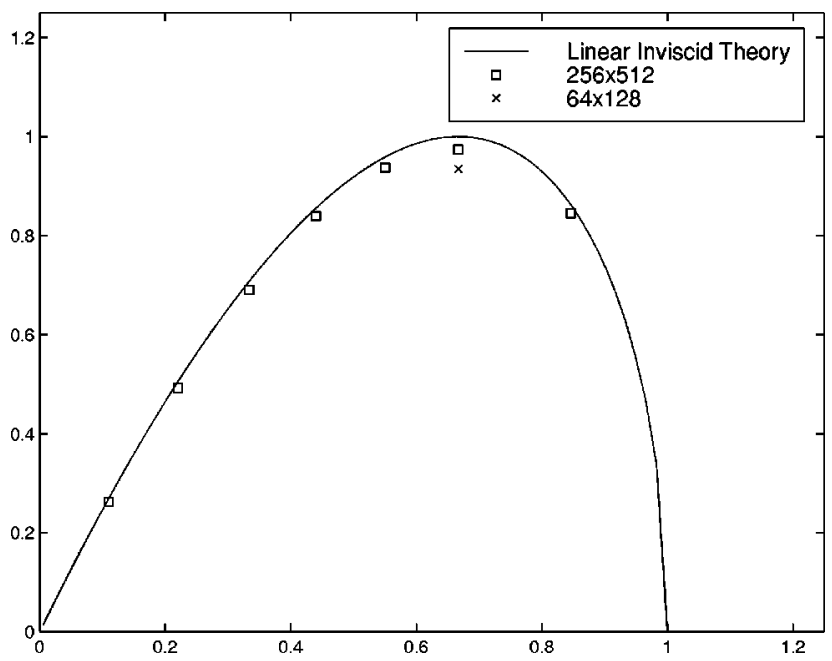

FIG. 3. Initial nondimensional growth rate for density ratio=10.0, $\operatorname{Re}_{1}=\operatorname{Re}_{2}=5000$; and various Weber numbers. 

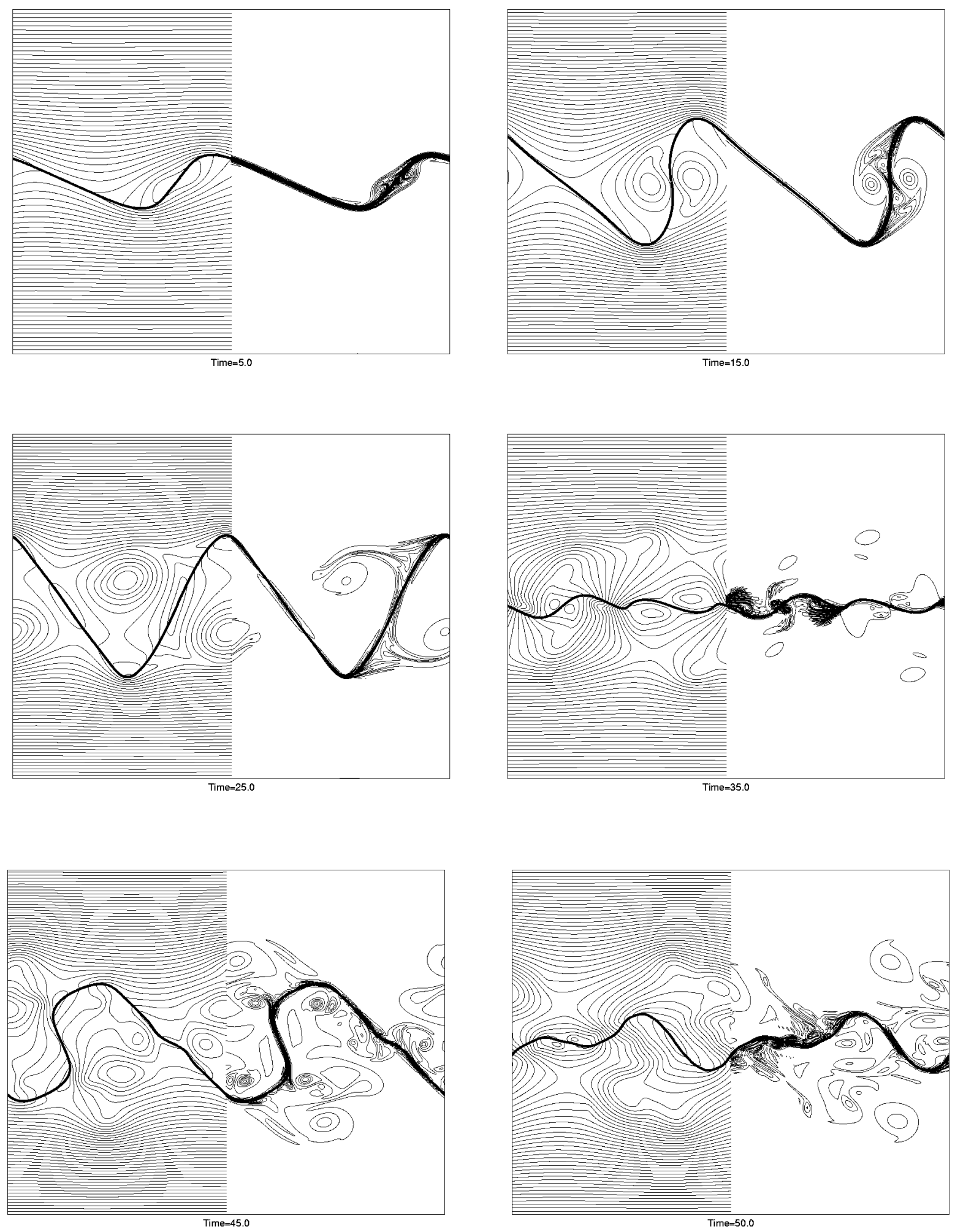

FIG. 4. Evolution for density ratio $=1.0, \mathrm{We}=3.0 ; \mathrm{Re}_{1}=\mathrm{Re}_{2}=10000$.

number simulations, viscous effects eventually lead to an increased thickness of the shear layer and therefore a reduction in the local shear strength. In addition to increased shear layer thickness by viscous diffusion, separation of vorticity from the crest of the wave, as seen at times 15.0 and 25.0, reduces the local pressure minimum above the crest. As the driving mechanism for the instability is removed, surface tension pulls the interface back and at $\tilde{t}$
$=35.0$, the interface has returned to a nearly flat position. The wave then grows again and is again pulled back to a nearly flat position, generating considerable amount of small-scale vorticity.

In Fig. 5, the Weber number has been increased to 6.0. The initial evolution is similar to the previous case, but at larger amplitude the wave folds over, resulting in a "finger" of one fluid penetrating into the other fluid. Similar "fingers" 

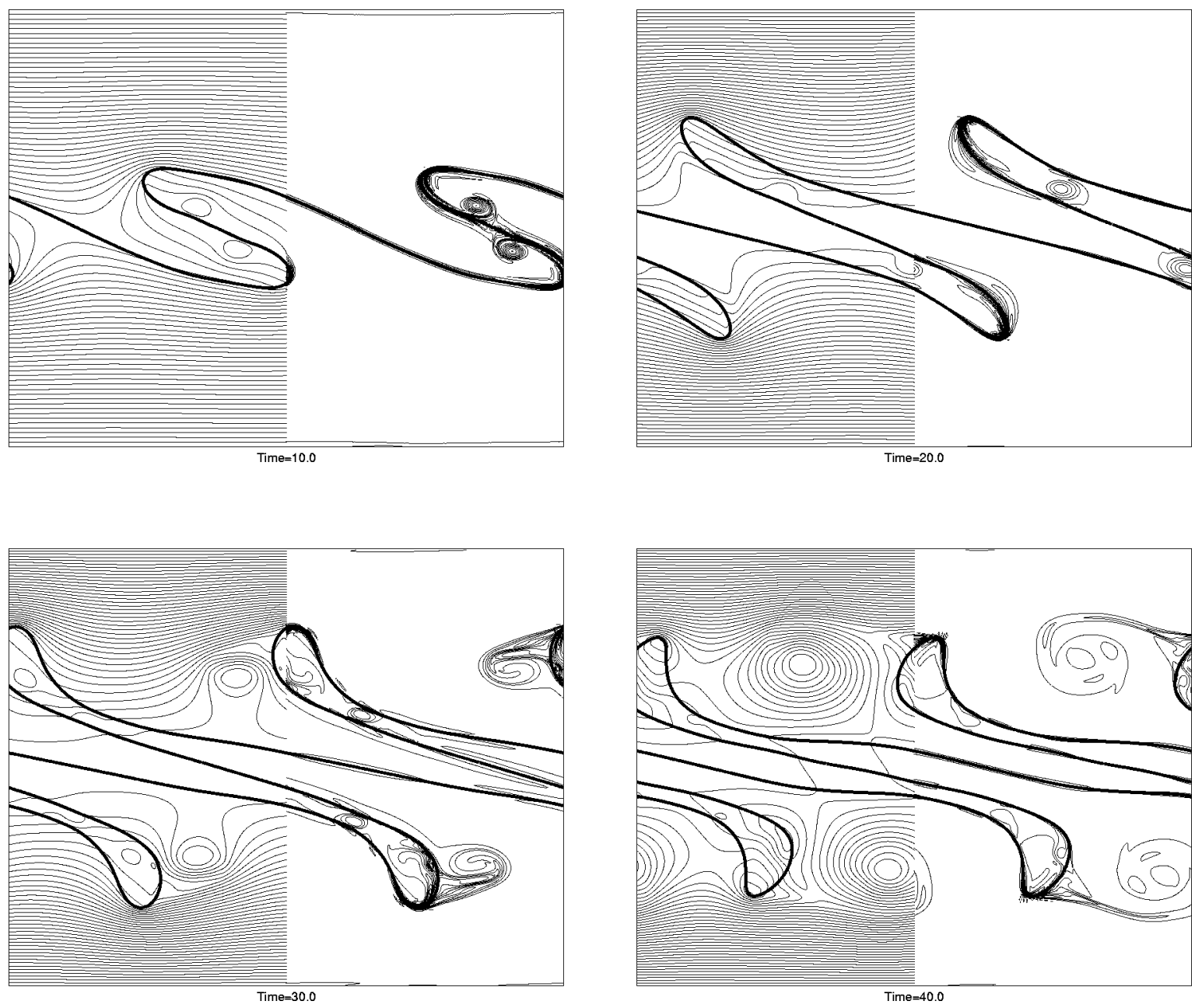

FIG. 5. Evolution for density ratio=1.0, $\mathrm{We}=6.0 ; \mathrm{Re}_{1}=\mathrm{Re}_{2}=10000$.

have also been seen by Hou et l. $^{7}$ The vortices that were initially shed from the crest of the wave are left behind as the finger grows. These vortices are relatively weak and dissipate quickly. At later times, the tips bend backwards and vorticity is again shed from the tip of the fingers, as seen at times 30.0 and 40.0. These shed vortices are much stronger than the vortices that formed initially, as seen from the deformation of the stream lines. The interface grows to a larger amplitude than for $\mathrm{We}=3.0$, due to the weaker effect of surface tension. As the shear layer becomes thicker, due to both diffusion and shedding of vorticity, surface tension eventually pulls the interface back, depositing vorticity into both fluids. Although most of the vorticity remains of one sign, a little bit of vorticity of the opposite sign is generated as surface tension effects become important. See, for example, the inward tip of the fingers at time 40 .

When the Weber number is increased to 10.0, see Fig. 6, the length of the fingers increases and the slope at which they penetrate into the other fluid decreases. The highest Weber number fingers are therefore nearly parallel to the interface. As the fingers pull back, fluid accumulates at the tip of each finger, forming a drop. This drop is connected to the rest of the finger by a thin thread that appears to have collapsed completely in some cases. While somewhat reminiscent of the formation of drops from a round ligament, the two dimensionality of this problem makes the usual explanation, that the azimuthal component of the surface tension causes the collapse of the ligament, not applicable. The formation of a thick border or a "two-dimensional drop" at the edge of a thin sheet of fluid has been examined in some detail by Song and Tryggvason. ${ }^{22}$ They showed that the border retreats with a constant velocity (relative to the ambient fluid) and that the thickness of the neck connecting the thick border with the rest of the sheet reaches a nearly constant value that decreases with the viscosity of the sheet. The neck, however, does not appear to reach a zero thickness and break for finite values of the viscosity.

As the Weber number is increased further to 15.0 , see Fig. 7, the initial wave folds over once before starting to be drawn out into a finger parallel to the interface. When the interface folds over a fluid drop appears to "pinch-off" near the fold, as seen at time $=20.0$. This pinch-off was also seen by Hou $\mathrm{et} \mathrm{al.}^{7}$ who studied the evolution up to the pinch-off time in great detail. Although the geometry is different from the formation of a drop at the end of a sheet, it is likely that the thickness of the thin layer only becomes zero in the inviscid limit. The interface is therefore not allowed to change its topology in the simulations and actual pinch-off does not 

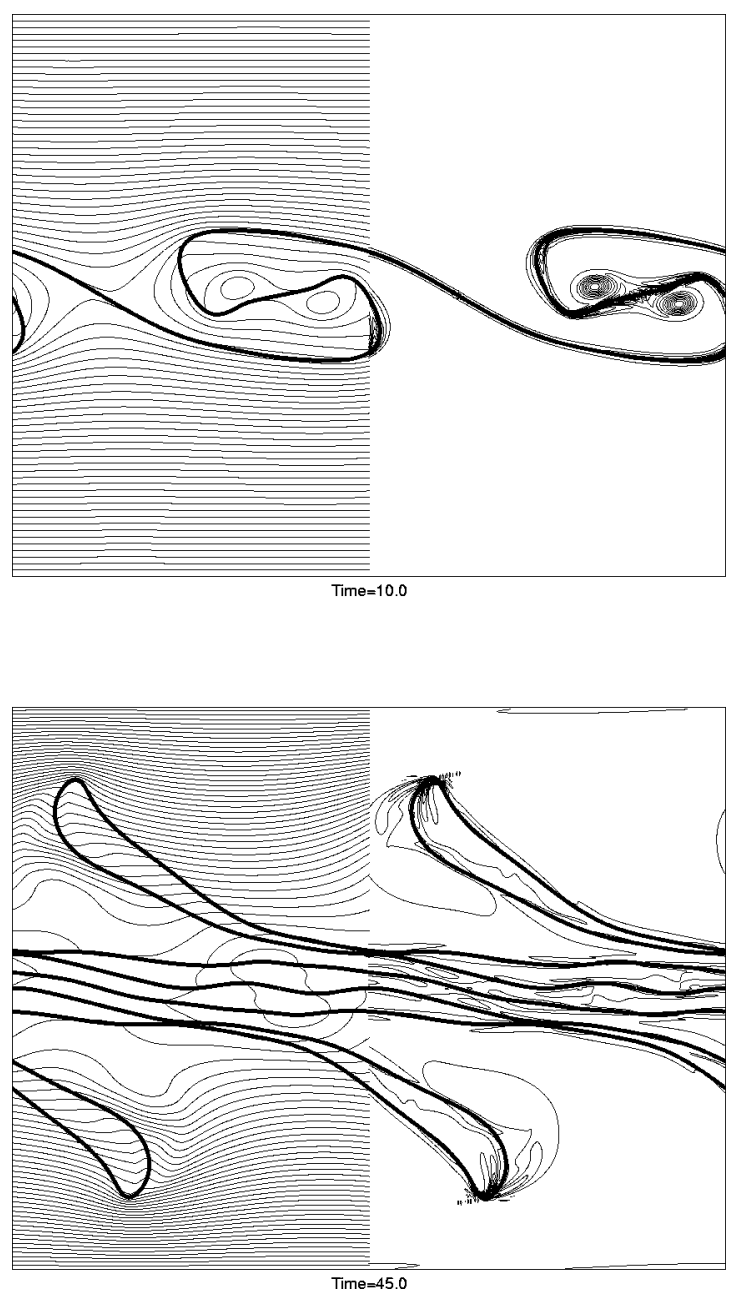

FIG. 6. Evolution for density ratio $=1.0, \mathrm{We}=10 ; \mathrm{Re}_{1}=\mathrm{Re}_{2}=10000$.

take place. As the folded fingers are stretched by the shear, the interface unwinds and a long wavy finger grows and then decays as before. At even higher Weber numbers, the interface rolls up as it would do if the fluids were miscible. We have not examined the transition from the folding-over to roll-up, but refer the reader to Hou et al. ${ }^{7}$ for inviscid simulations in the higher Weber number range and an examination of the "pinch-off." At very high Weber numbers the interface is less "stiff" and separation less pronounced. We therefore expect their results to give a very good picture of the evolution of real systems in that parameter range.

\section{Nonlinear evolution for a density ratio of $\mathbf{1 0}$}

The evolution of an interface separating fluids with a density ratio of 10 and a Weber number equal to 1.7 is shown in Fig. 8. The corresponding wavelength is close to the linear most unstable wave $\left(\mathrm{We}_{\max }=1.65\right)$. The initial conditions are derived from a normal mode analysis, as in the zero density difference case. The initial amplitude $\zeta$ is equal to $5 \%$ of the wavelength and $\operatorname{Re}_{1}=5000$ (heavy fluid), and $\operatorname{Re}_{2}=1000$ (light fluid). The Reynolds numbers used here are smaller than those used for the zero density difference case to ensure that the boundary layer on either side of the interface is well resolved. As we saw for the smaller density ratio,
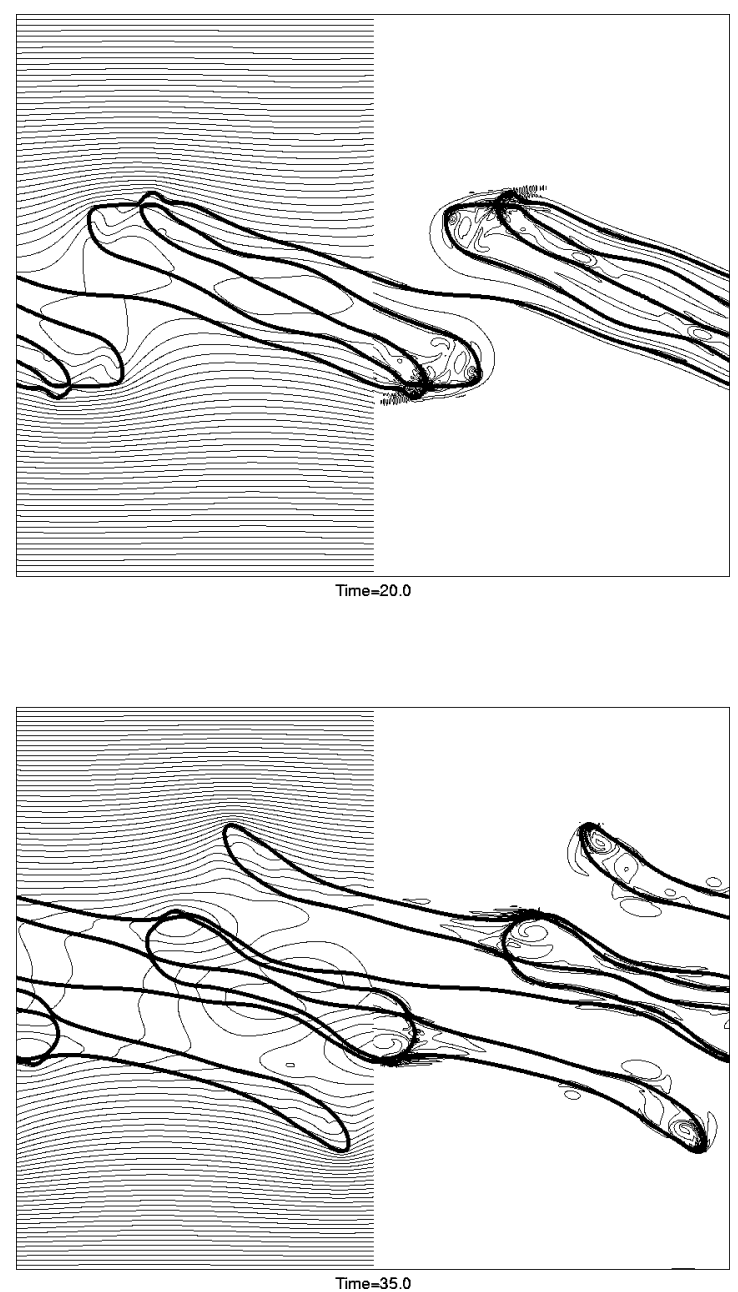

FIG. 7. Evolution for density ratio=1.0, $\mathrm{We}=15.0, \mathrm{Re}_{1}=\mathrm{Re}_{2}=10000$.

the initial perturbation grows and the wave becomes steeper. Here, however, the vorticity is advected to the tip of the wave crest where the heavy fluid reaches into the lighter fluid, and the wave is asymmetric with a smooth trough and a sharp crest. The wave moves to the right, with the heavy bottom fluid, as the left side becomes steeper. There is a strong asymmetry in the vorticity field as well, and almost all the vorticity is shed from the wave crest into the lighter fluid. The strong deposition of vorticity in the lighter fluid is due to two effects. First, the kinematic viscosity of the lighter fluid is higher, so vorticity diffusion into it is more rapid than into the heavier fluid. Second, the curvature of the finger penetrating in the lighter fluid is higher than of the one extending into the heavier fluid, and this leads to stronger vorticity production. For this Weber number, surface tension quickly stabilizes the wave and we see again that waves close to the linear most unstable wavelength grow initially, but viscous effects prevent them from reaching large amplitudes.

The evolution for a Weber number equal to 5.0 is shown in Fig. 9. Again there is an asymmetry in the evolution of the interface and the vorticity, as the wave becomes steeper, and eventually a thin finger, or a ligament, of heavy fluid is pulled from the crest into the lighter fluid. Diffusion and shedding of vorticity from the crest eventually reduces the 

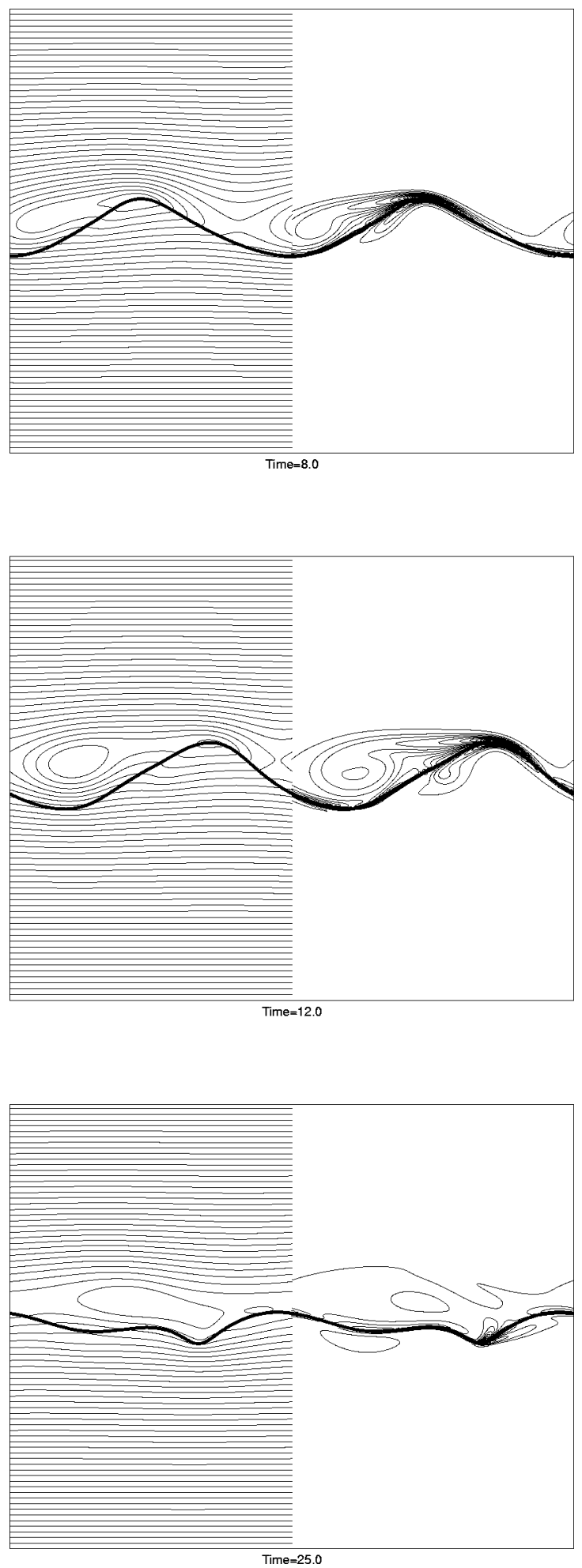

FIG. 8. Evolution for density ratio=10.0, We=1.7, $\operatorname{Re}_{1}=5000 ; \mathrm{Re}_{2}$ $=1000$.

shear and surface tension begins to pull the finger back. As the finger is pulled back, a circular drop is formed at its tip.

In Fig. 10, we show results for a higher Weber number, $\mathrm{We}=10.0$. The evolution is similar to the $\mathrm{We}=5.0$ case, but the fingers become longer and narrower as the Weber number increases. This parallels the results for the zero density dif- ference. Once the fingers start to retreat, we see again the formation of two-dimensional drops.

Even though the density ratio is only ten here, the evolution is much more reminiscent of what we would expect to see for an air/water interface, for example, than the zero density difference case. The reason is the advection of vorticity along the interface by the density weighted velocity. In the limit of a completely free surface, the vorticity simply moves with the heavy fluid. For inviscid flows, the interface vorticity is always bound to the interface, but for viscous flows we generally expect separation, particularly for a nonzero surface tension.

The resolution used for the computations presented here has been selected so that reasonably high Reynolds numbers could be simulated in a reasonable amount of time. To assess the adequacy of the resolution used, several grid refinement studies have been carried out. Figure 11 shows the interface at time 30 for the case shown in Fig. 9, computed on both a 128 by 256 and a 256 by 512 grid. Obviously, the results are very similar. The only difference is that the better resolved finger is slightly shorter. At this stage the fingers are being pulled back by surface tension and since the interface is smoothed out more on the coarsest grid, the effect of surface tension is slightly weaker.

\section{Discussion}

To understand the results presented in the preceding sections better, we have examined the data in a number of ways. In Figs. 12 and 13, the peak-to-peak amplitude, normalized by the wavelength, is shown versus the nondimensional time for various Weber numbers. Figure 12 shows the results for a zero density difference. Initially, the growth of the wave is extremely rapid as expected from linear theory. For We $=6.0,10.0$, and 15.0 the nondimensional amplitudes are nearly the same for times less than 5.0, since the nondimensional growth rate becomes linearly proportional to the wave number in the limit of large Weber numbers and therefore independent of the Weber number, see Eq. (3). For smaller Weber numbers the growth rate is no longer linearly proportional to the wave number and the growth rate is smaller. At later times, the growth rate slows down when nonlinear and viscous effects take over. For $\mathrm{We}=3.0$, the amplitude oscillates as the initially growing wave becomes a standing capillary wave. As the Weber number is increased, the maximum amplitude increases until roll-up begins to occur. A comparison between the graph in Fig. 12 and Fig. 5 shows that the amplitude starts to decay as the fingers stop to grow. In Fig. 13, the amplitude is plotted versus time for $r=10$. The growth rate of the most unstable wave reaches its maximum amplitude quickly. While the amplitude for the $\mathrm{We}=5.0$ case stops growing around time 20, when a distinct drop has formed at the tip of the fingers, the amplitude grows slightly again due to the continuing increase in the depth of the trough where the light fluid reaches into the heavier fluid (see Fig. 9). As the Weber number is increased to 10.0, we see that the maximum amplitude also increases.

To examine the effect of viscosity, the peak-to-peak amplitude for $\operatorname{Re}_{1}=\operatorname{Re}_{2}=10000, r=1, \mathrm{We}=3.0$ is compared to 

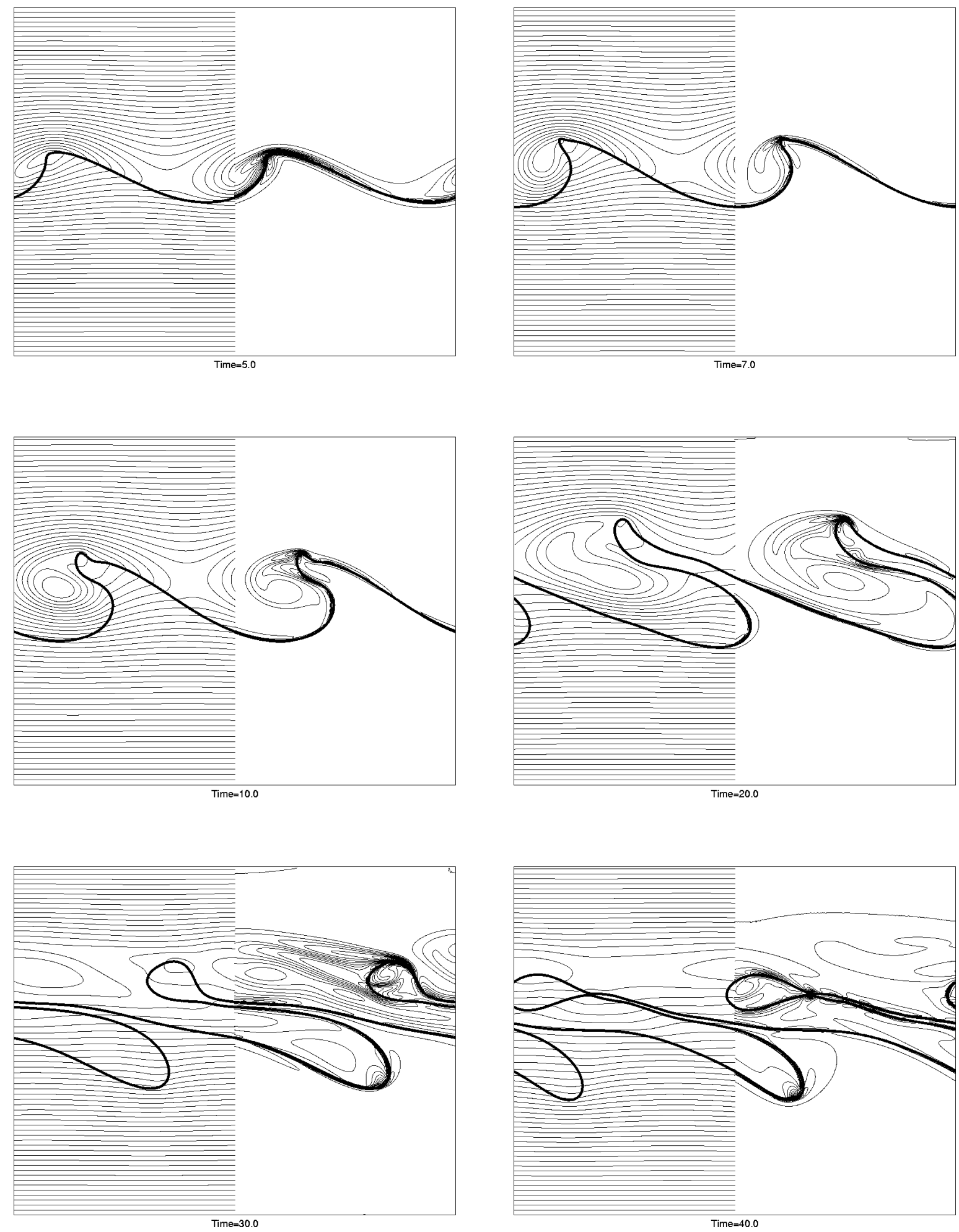

FIG. 9. Evolution for density ratio=10.0, We=5.0; $\operatorname{Re}_{1}=5000 ; \mathrm{Re}_{2}=1000$.

the case of $\operatorname{Re}_{1}=\operatorname{Re}_{2}=5000$ in Fig. 14. For these Reynolds numbers, the initial growth rates are similar, since viscous effects are negligible at early times. However, at later times and larger amplitudes viscous effects begin to become important. The amplitude of the smaller Reynolds number wave begins to decay at an earlier time after reaching a smaller maximum amplitude. This trend is continued in the second growth-and-decay phase where the amplitude of the interface oscillations decrease with the Reynolds number. While the maximum amplitude of the interface depends on the Reynolds number, the overall evolution of the instability is not significantly changed as long as the Reynolds numbers are sufficiently large.

The average horizontal velocity profile, computed by av- 

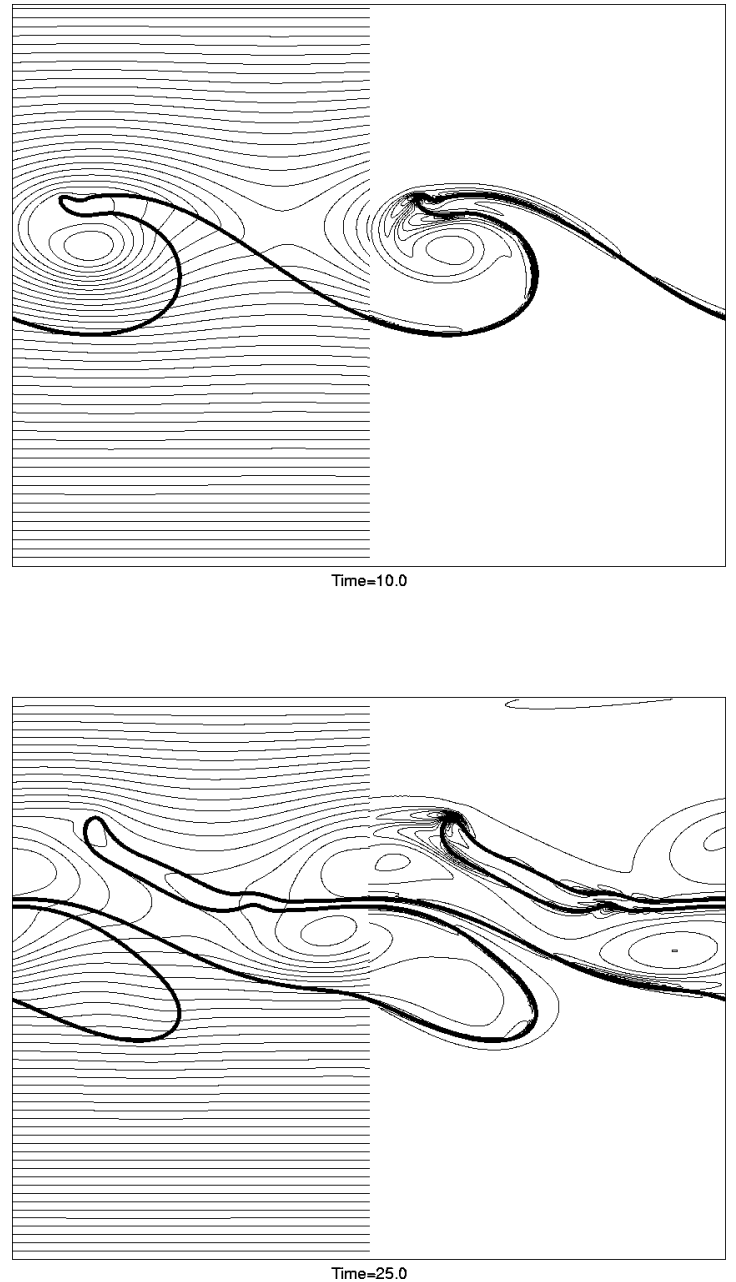

FIG. 10. Evolution for density ratio=10.0, We=10.0; $\operatorname{Re}_{1}=5000 ; \mathrm{Re}_{2}$ $=1000$.

eraging over horizontal grid lines, is plotted for two different cases and several different times in Figs. 15 and 16. In Fig. 15 , where $\mathrm{We}=6.0$ and $r=1$, the velocity profile grows symmetrically about the original flat interface. Initially, the width of the shear layer grows rapidly, but the velocity profile remains approximately linear. At later times, it stops spreading and develops an " $N$ " shape due to flow reversal caused by the massive separation of vorticity from the tip of the fingers. Indeed, Fig. 5 shows that the shear layer consists of two rows of primary vortices, even though the initial conditions contained only one wavelength. In Fig. 16, where $r=10$ and

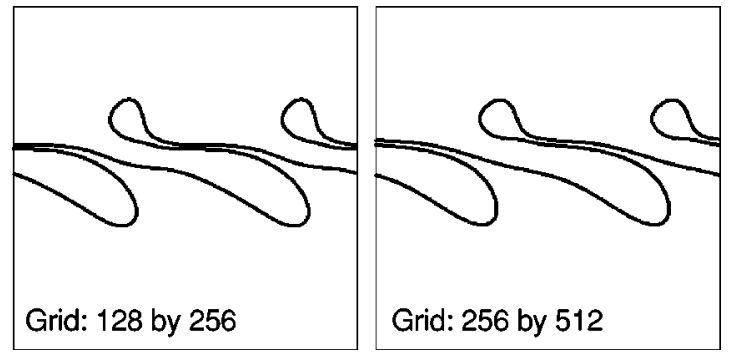

FIG. 11. Grid resolution test for the case shown in Fig. 9. Here $\mathrm{We}=5, r$ $=10, \mathrm{Re}_{1}=5000$, and $\mathrm{Re}_{2}=1000$.

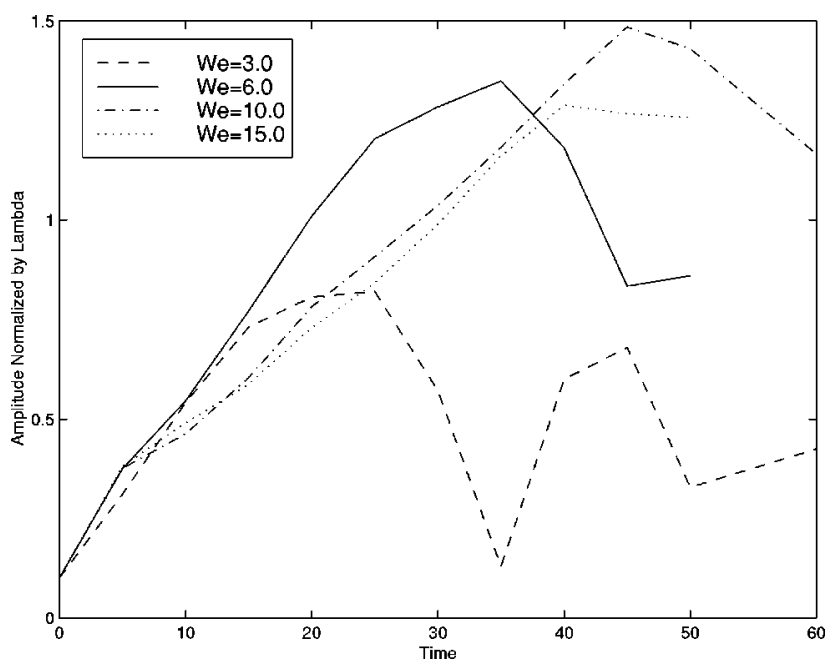

FIG. 12. Amplitude vs time for density ratio=1.0.

$\mathrm{We}=5.0$, the asymmetry of the interface is reflected in the evolution of the velocity profile. The velocity of the lighter fluid decreases significantly, but the velocity of the heavy fluid remains nearly unchanged. Again, we see a rapid increase in the thickness of the shear layer initially and flow reversal at late times when the rate of growth is slower. At early time, the velocity profile over-shoots, as separation of vorticity from the crest of the wave speeds up the velocity in the trough (see the streamlines in Fig. 9 at times 7 and 10). The slight "hump" in the velocity profile at the latest time is the result of the finger of heavy fluid being pulled to back by surface tension.

To quantify the thickness of the shear layer, we use the moments of the velocity profile gradient defined by

$$
M_{n}=-\int_{-\lambda}^{+\lambda} y^{n} \frac{d U_{\text {ave }}}{d y} d y \quad \text { for } \quad n=0,1,2, \ldots .
$$

Here, $U_{\text {ave }}$ is the velocity averaged in the horizontal direction, and $y=0$ is the original location of the flat interface. The zeroth moment is simply the difference in the velocity

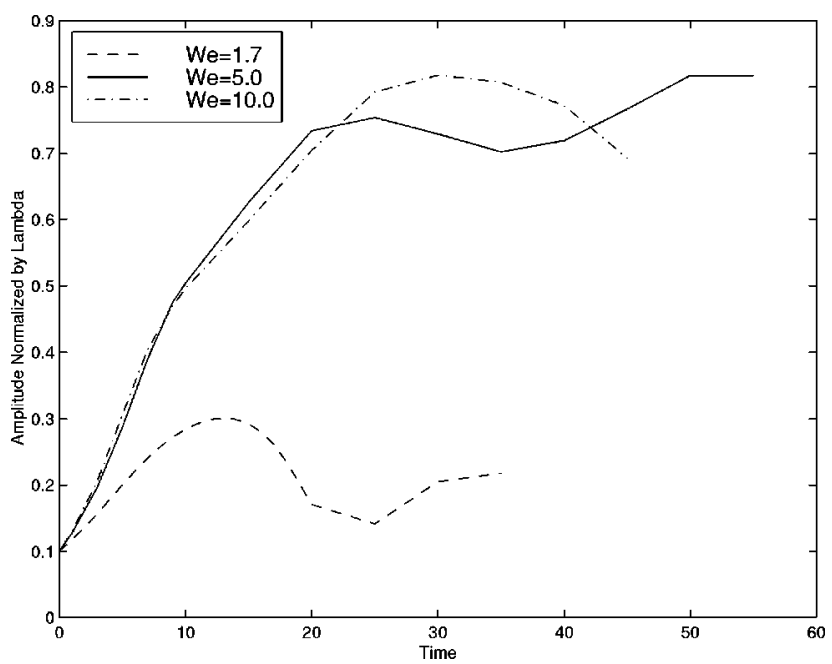

FIG. 13. Amplitude vs time for density ratio=10.0. 


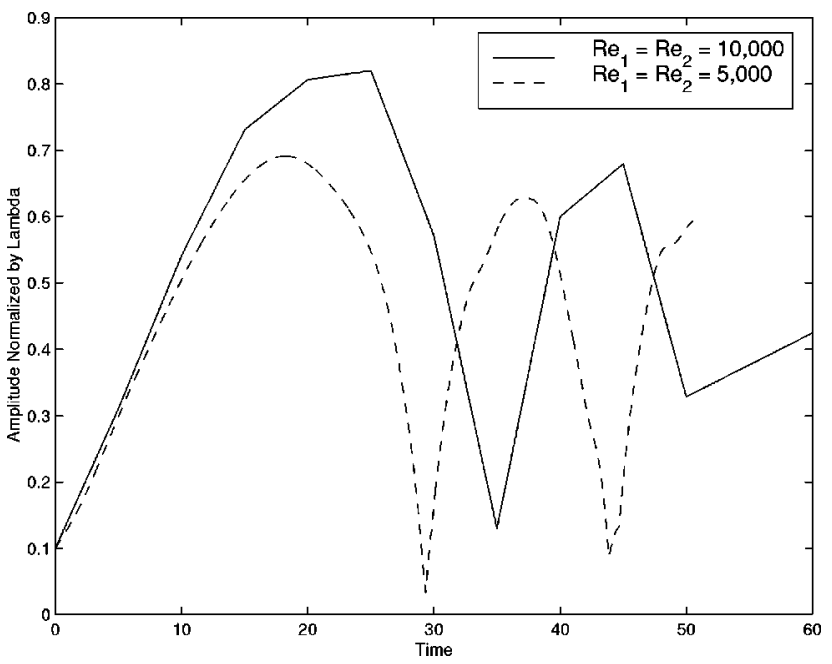

FIG. 14. Amplitude vs time for density ratio=1.0, We=3.0; $\mathrm{Re}_{1}=\mathrm{Re}_{2}$ $=10000$, and $\operatorname{Re}_{1}=\operatorname{Re}_{2}=5000$.

above and below the interface, $M_{0}=U_{1}-U_{2}$; the first moment measures the skewness of the velocity gradient, and the second moment yields the width of the shear layer. The boundary displacement thickness used for wall bounded shear flows cannot be used here, because its value depends on which frame of reference one chooses.

The value of the moments as defined by Eq. (19) depends on where we set $y=0$. To avoid this ambiguity, we define a new origin by

$$
y=\Delta+y^{\prime},
$$

where $\Delta$ is selected in such a way that the first moment about $y^{\prime}$ is zero. Substituting for $y$ in the definition of the first moment [Eq. (19) with $n=1$ ] yields

$$
M_{1}=-\int_{-\lambda}^{+\lambda}\left(y^{\prime}+\Delta\right) \frac{d U_{\text {ave }}}{d y} d y=M_{1}^{\prime}+\Delta M_{0} .
$$

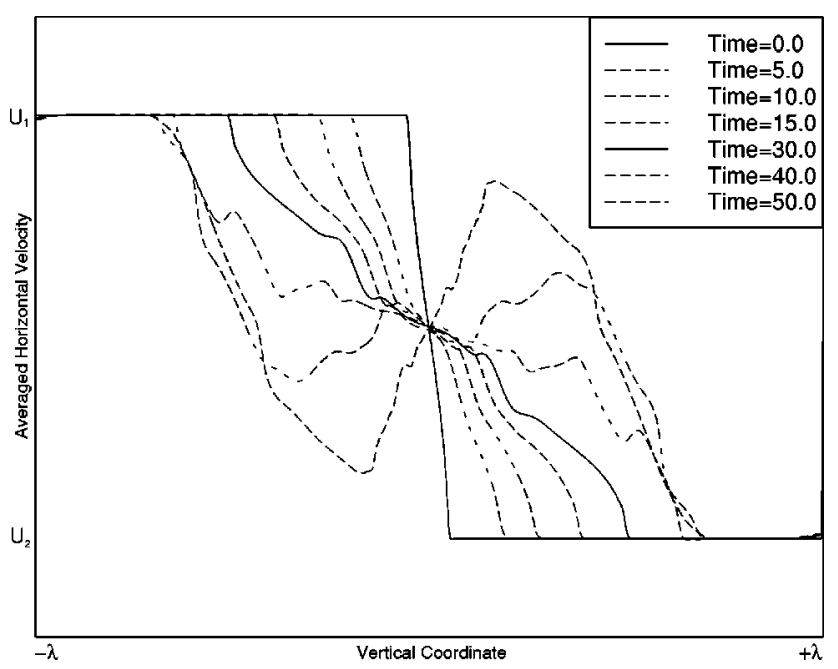

FIG. 15. Averaged horizontal velocity for density ratio=1.0, We=6.0; $\operatorname{Re}_{1}=\operatorname{Re}_{2}=10000$.

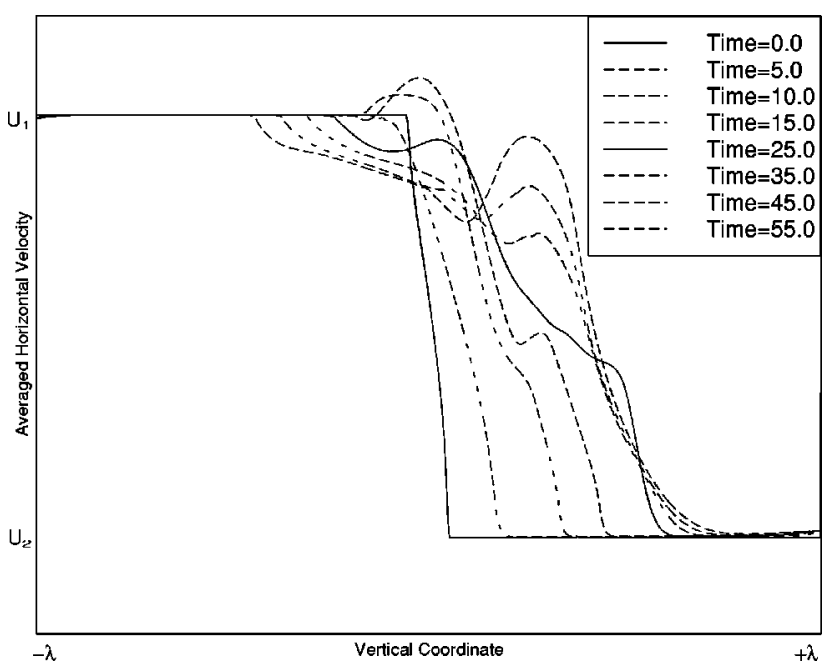

FIG. 16. Averaged horizontal velocity for density ratio $=10.0, \mathrm{We}=5.0$; $\operatorname{Re}_{1}=5000 ; \operatorname{Re}_{2}=1000$.

Selecting $\Delta=M_{1} / M_{0}$ makes $M_{1}^{\prime}=0$ and the second moment around the new origin is easily found: $M_{2}^{\prime}=M_{2}-\Delta^{2} M_{0}$.

For zero density stratification, $\Delta$ is zero for all times and Weber numbers, since the velocity profile remains symmetric about $y=0$. For a finite density ratio, on the other hand, most of the change in the velocity takes place in the lighter fluid and $\Delta$ is positive. In Fig. 17, the nondimensional displacement, $\widetilde{\Delta}=\Delta / \lambda$ is plotted versus time for three Weber numbers. For $\mathrm{We}=5$ and 10 the displacement increases until it saturates at about a third of the wavelength, but for We $=1.7$, the growth is less and the displacement saturates at about $10 \%$ of the wavelength.

The second moment, $M_{2}^{\prime}$, is plotted versus time in Figs. 18 and 19 for all the Weber numbers simulated. In Fig. 18, where $r=1$, the second moments are approximately the same at early times. However, around $t=10.0, M_{2}^{\prime}$ is greater for $\mathrm{We}=3.0$ and 6.0 than for $\mathrm{We}=10.0$ and 15.0. This can be explained by looking at the peak-to-peak amplitudes in Fig. 12. For $t>10.0$, the amplitude for the low Weber number cases are larger than those of the higher Weber number ones,

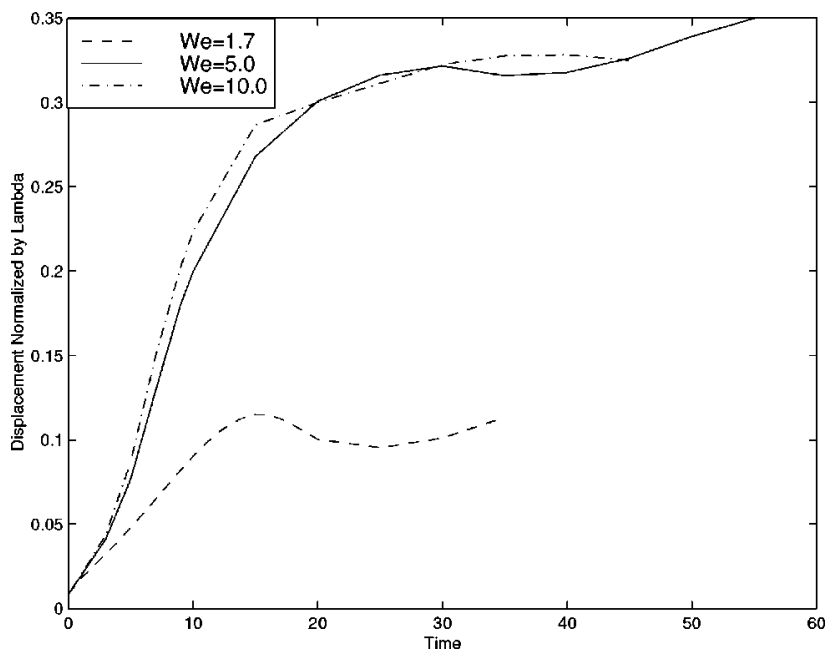

FIG. 17. Displacement vs time for density ratio=10.0. 


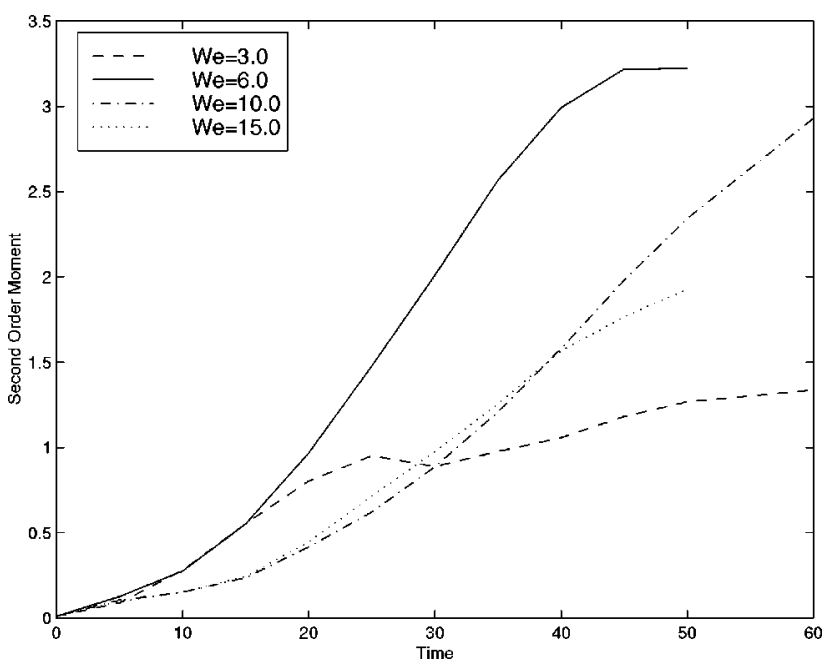

FIG. 18. Second order moment vs time for density ratio $=1.0$.

since the slopes at which the fingers penetrate into each fluid are larger for the smaller Weber numbers. Therefore, the velocity deficit due to vorticity shed from the fingers occurs further away from $y=0$, resulting in a larger $M_{2}^{\prime}$. When the amplitude of the interface stops growing, $M_{2}^{\prime}$ continues to grow due to diffusion, but at a slower rate. In Fig. 19, $M_{2}^{\prime}$ is plotted versus time for $r=10$. Initially, $M_{2}^{\prime}$ increases, but then $M_{2}^{\prime}$ actually decreases between times 7 and 15 , due to the overshoot in the velocity profile.

The effect of viscosity can also be seen by examining the energy. While the surface energy increases initially at the expense of kinetic energy, the total energy must decrease due to dissipation. In Fig. 20 we show the total energy

$$
E_{\mathrm{tot}}=T \cdot S+\int_{V} \frac{1}{2} \rho \mathbf{u} \cdot \mathbf{u} d a
$$

for the $r=1$ simulations. Here $S$ is the interface length and

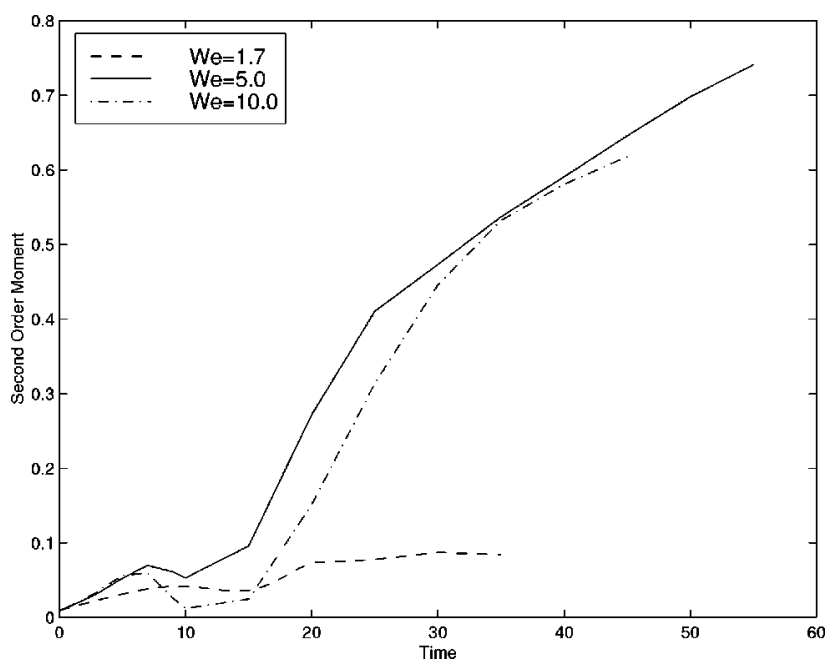

FIG. 19. Second order moment vs time for density ratio $=10.0$.

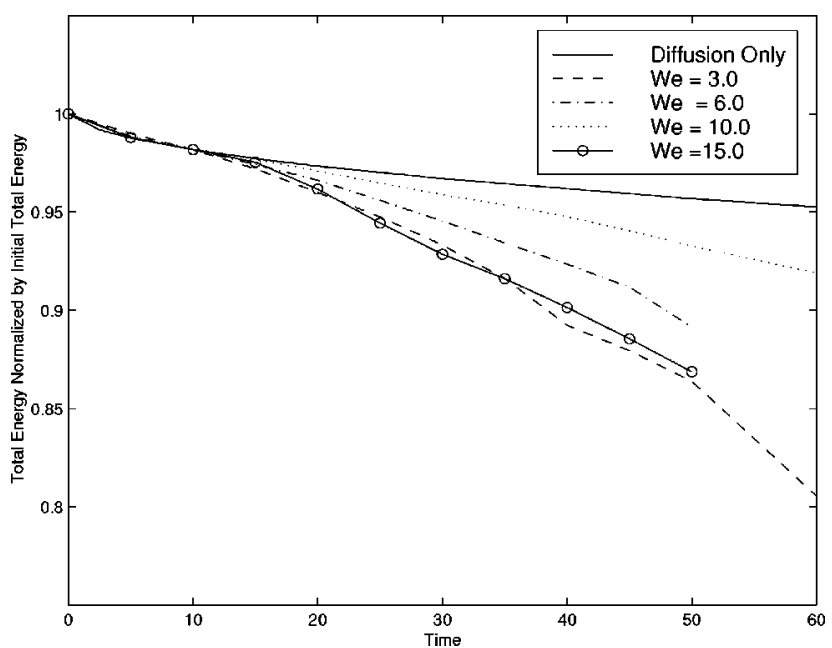

FIG. 20. Total energy for density ratio=1.0.

the integral is over the whole computational domain. The energy has been normalized by the total initial energy. For reference we also include the total energy for a completely flat interface, calculated using the solution to Stokes' first problem,

$$
u(y)=U \operatorname{erf}\left(\frac{y}{2 \sqrt{\nu t}}\right) .
$$

Initially the total energy decreases at about the same rate in all cases. However, after time $=15.0$, the vortex shedding increases the energy dissipation. The energy dissipation rate decreases with increasing Weber numbers for $\mathrm{We}=3.0$ to 10.0 , but for $\mathrm{We}=15.0$, where the interface starts to roll-up the rate increases again. In Fig. 21 where the total energy is plotted versus time for $r=10.0$, the energy losses for We $=5.0$ and 10.0 are essentially the same. The loss is smaller for $\mathrm{We}=1.7$ since the instability grows only slightly before it

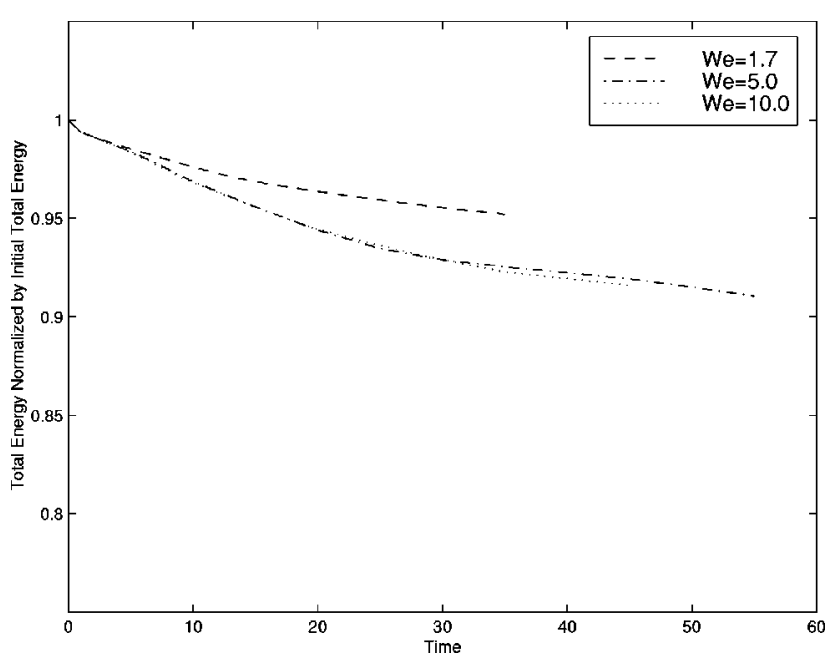

FIG. 21. Total energy for density ratio $=10.0$. 

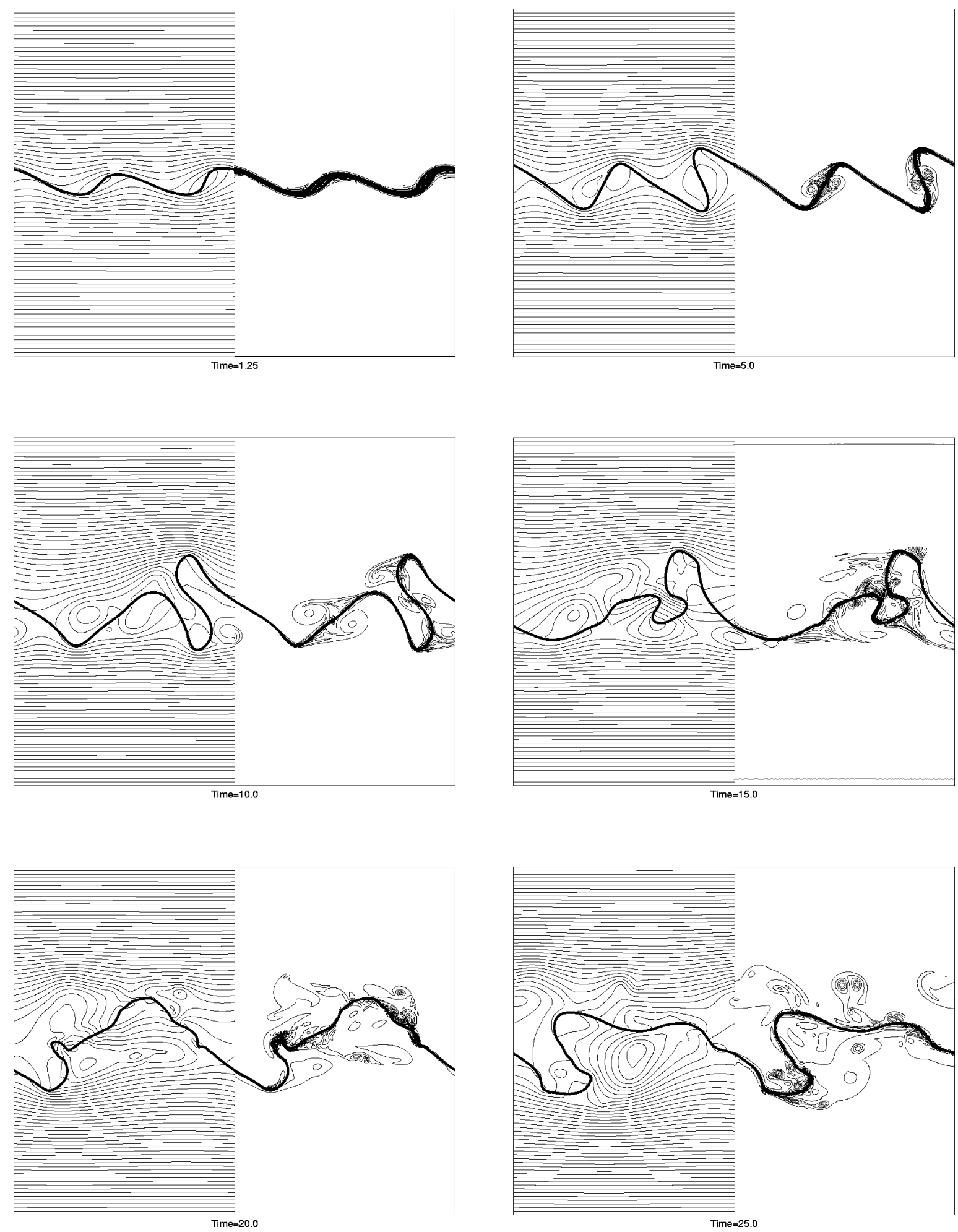

FIG. 22. Evolution of two mode initial conditions. Density ratio=1.0, We $=6.0 ; \operatorname{Re}_{1}=\operatorname{Re}_{2}=10000$.

begins to decay and there is little vortex shedding. For larger Weber numbers the dissipation due to vortex shedding is greater, resulting in a greater rate of energy loss. The nonmonotonic behavior of the dissipation versus Weber number is similar to the results of Yang and Tryggvason ${ }^{23}$ for the microbreaking of interfacial waves.

\section{E. Nonlinear evolution of two modes}

The results in preceding sections show that while the inviscidly most unstable linear wave grows initially, it is quickly stabilized. Longer waves, on the other hand, continue to grow. This suggests that initial conditions with both 
short and long waves will lead to a competition where the short waves first do better but the long waves eventually win. In Fig. 22 we show the evolution of an interface where the initial perturbation consists of a linear addition of two waves,

$$
y(x)=\frac{3}{4} \zeta \cos \left(\frac{2 \pi x}{\lambda_{\text {short }}}\right)+\frac{1}{4} \zeta \cos \left(\frac{2 \pi x}{\lambda_{\text {long }}}\right) .
$$

The interface and the flow field is shown at six evenly spaced times (with the exception of the first frame). Two periods are shown, with the vorticity plotted on the right and the stream function on the left. Here $\zeta$ is $5 \%$ of the longer wavelength. The Weber number based on the shorter wave is equal to 3.0, which is the linearly most unstable wave. The Weber number based on the longer wave is $\mathrm{We}=6.0$ and its amplitude is one-third of the shorter wave. Other parameters are $\operatorname{Re}_{1}$ $=\operatorname{Re}_{2}=10000$, based on the longer wavelength, and $r=1$. Since the shorter wave is the most unstable one, it initially grows rapidly. At later times however, when vorticity is being shed from the crests of the waves and viscous effects are important, the amplitude of the shorter wave decays and the long wave begins to grow. This agrees with our results for just one wave, where longer waves grew to larger amplitudes. As the short wave is stabilized, large amounts of small scale vorticity is generated. This vorticity further increases the growth of the shear layer, accelerating the stabilization of the shorter wave.

\section{CONCLUSION}

Several simulations of the two-dimensional KelvinHelmholtz instability of immiscible fluids are presented. The Reynolds numbers are selected to be sufficiently high so that the initial instability is well predicted by inviscid linear stability theory. At larger amplitudes, however, viscous effects become important. The viscous effects are manifested both by diffusive growth of the shear layer, as well as shedding of vorticity. The increased thickness results in a reduction of the strength of the inertial mechanism driving the growth of the instability and allows surface tension to stabilize the inviscidly most unstable wavelength. While the growth of the initially most unstable wave is saturated, longer waves continue to grow.

We have conducted two sets of simulations, one for a zero density difference between the fluids on either side of the shear layer and the other one for a density ratio of 10. For zero density differences the evolution is symmetric with respect to the different fluids. However, surface tension prevents Kelvin-Helmholtz roll-up as seen for miscible fluids and fingers of one fluid penetrate the other. The slope of these fingers depends on the nondimensional wavelength (Weber number). While viscous effects limit the growth of these fingers at low Weber numbers, high Weber number fingers can become very long. When the interface growth is stabilized by viscous effects, considerable amount of small scale vorticity is generated, further increasing the thickness of the shear layer. At even higher Weber numbers, the interface starts to exhibit a behavior more similar to the classical nonlinear Kelvin-Helmholtz instability and roll-up. The transition is complex, however, and we find intermediate states where the interface folds over once before being stretched into a long finger, for example.

At larger density ratios, the evolution is no longer symmetric and waves of the heavy fluid grow into the lighter one. As for the zero density difference, waves with wavelengths close to the most unstable one are generally stabilized at large amplitude by viscous effects but longer wavelengths lead to a "wave breaking" where a finger of the heavy fluid is pulled into the lighter fluid by the effect of the shear. Two-dimensional "drops" form at the tips of these fingers of heavy fluid.

Simulations of two initial modes (the inviscidly most unstable one and another one twice as long) show that while the short wave grows initially, it is the longer wave that eventually dominates. The transition is, however, fairly complex and considerable amount of small scale vorticity is generated during the selection process.

\section{ACKNOWLEDGMENTS}

Effort sponsored by the Air Force Office of Scientific Research, Air Force Material Command, USAF, under Grants Nos. F49620-96-1-0356 and F49620-97-1-0525. The U.S. Government is authorized to reproduce and distribute reprints for Governmental purpose notwithstanding any copyright notation thereon. The views and conclusions contained herein are those of the author and should not be interpreted as necessarily representing the official policies or endorsements, either expressed or implied, of the Air Force Office of Scientific Research or the U.S. Government.

${ }^{1} \mathrm{~L}$. Rosenhead, "The formation of vortices from a surface of discontinuity,” Proc. R. Soc. London, Ser. A 134, 170 (1931).

${ }^{2}$ R. Kransy, "Desingularization of periodic vortex sheet roll-up," J. Comput. Phys. 65, 292 (1987).

${ }^{3}$ P. C. Patnaik, F. S. Sherman, and G. M. Corcos, "A numerical simulation of Kelvin-Helmholtz waves of finite amplitude,” J. Fluid Mech. 73, 215 (1976).

${ }^{4}$ G. M. Corcos and F. S. Sherman, "The mixing layer: Deterministic models of turbulent flow. Part I. Introduction and the two-dimensional flow," J. Fluid Mech. 139, 29 (1984).

${ }^{5}$ G. Tryggvason, W. J. A. Dahm, and K. Sbeih, "Fine structure of vortex sheet roll-up by viscous and inviscid simulations," ASME J. Fluids Eng. 113, 31 (1991)

${ }^{6}$ R. W. Metcalfe, S. A. Orszag, E. Brachet, S. Menon, and J. J. Riley, "Secondary instability of a temporally growing mixing layer," J. Fluid Mech. 184, 207 (1987).

${ }^{7}$ T. Y. Hou, J. S. Lowengrub, and M. J. Shelley, "The long-time motion of vortex sheets with surface tension," Phys. Fluids 9, 1933 (1997).

${ }^{8} \mathrm{C}$. Porzikidis, "Instability of two-layer creeping flow in a channel with parallel-sided walls," J. Fluid Mech. 351, 139 (1997).

${ }^{9}$ B. Lafaurie, C. Nardone, R. Scardovelli, S. Zaleski, and G. Zanetti, "Modelling merging and fragmentation in multiphase flows with SURFER," J. Comput. Phys. 113, 134 (1994).

${ }^{10}$ A. Lefebvre, Atomization and Sprays (Taylor and Francis, Washington, DC, 1989).

${ }^{11}$ L. Bayvel and Z. Orzechowski, Liquid Atomization (Taylor and Francis, Washington, DC, 1993).

${ }^{12} \mathrm{Z}$. Farago and N. Chigier, "Morphological classification of disintegration of round liquid jets in a coaxial air stream," Atomization Sprays 2, 137 (1992)

${ }^{13}$ P.-K. Wu, R. F. Miranda, and G. M. Faeth, "Effects of initial flow conditions on primary breakup of nonturbulent and turbulent liquid jets," AIAA Pap. 94-0561 (1994).

${ }^{14}$ S. O. Unverdi and G. Tryggvason, “The shear breakup of an immiscible 
fluid interface," in Fluid Dynamics at Interfaces, edited by W. Shyy and R. Narayanan (Cambridge University Press, Cambridge, 1999).

${ }^{15} \mathrm{~W}$. Tauber and G. Tryggvason, "Direct numerical simulation of primary breakup,” Comp. Fluid Dynamics J. 9, 158 (2000).

${ }^{16}$ P. G. Drazin and W. H. Reid, Hydrodynamic Stability (Cambridge University Press, Cambridge, 1981).

${ }^{17}$ J. Adams, "MUDPACK: Multigrid Fortran software for the efficient solution of linear elliptic partial differential equations," Appl. Math. Comput. 34, 13 (1989).

${ }^{18}$ C. S. Peskin, "Numerical analysis of blood flow in the heart," J. Comput. Phys. 25, 220 (1977).

${ }^{19}$ G. Tryggvason, B. Bunner, A. Esmaeeli, D. Juric, N. Al-Rawahi, W.
Tauber, J. Han, S. Nas, and Y.-J. Jan, "A front tracking method for the computations of multiphase flow," J. Comput. Phys. 169, 708 (2001).

${ }^{20}$ M. R. Nobari, Y.-J. Jan, and G. Tryggvason, "Head on collision of drops-A numerical investigation," Phys. Fluids 8, 29 (1996).

${ }^{21}$ A. Esmaeeli and G. Tryggvason, "Direct numerical simulations of bubbly flows. Part I—Low Reynolds number arrays," J. Fluid Mech. 377, 313 (1998).

${ }^{22} \mathrm{M}$. Song and G. Tryggvason, "The formation of thick borders on an initially stationary fluid sheet," Phys. Fluids 11, 2487 (1999).

${ }^{23}$ Y. Yang and G. Tryggvason, "Dissipation of energy by finite amplitude surface waves," Comput. Fluids 27, 829 (1998). 